\title{
Complicações em implantodontia e prótese sobre implante: revisão de literatura
}

Complications in implantology and implant for implants: literature review

Complicaciones de implantología y prótesis de implantes: revisión de la literatura

Diana Leite BATISTA

Cirurgiã Dentista. Especialista em Implantodontia. Centro Educacional Dental Press, 87015-001 Maringá-PR, Brasil

Rodrigo Lorenzi POLUHA

Departamento de Odontologia, Universidade Estadual de Maringá -UEM. 87080-000 Maringá-PR, Brasil https://orcid.org/0000-0001-7180-6448

\section{Resumo}

Desde que a utilização sistemática de implantes como possibilidade terapêutica eficaz foi comprovada, a busca por esse tratamento pelos pacientes com edentulismo foi ampliada. Ainda assim, como qualquer intervenção em um organismo vivo, o tratamento reabilitador através do uso de implantes apresenta possíveis complicações, tanto em relação aos implantes quanto as reabilitações implanto-suportadas. Por mais que o correto planejamento não impeça a ocorrência dessas falhas e complicações no tratamento implantoprotético, permitirá ao profissional uma previsibilidade desses intercorrências, possibilitando uma conduta eficiente a cada caso.

Descritores: Implantes Dentários; Próteses e Implantes; Complicações.

\section{Abstract}

Since the systematic use of implants as an effective therapeutic possibility has been proven, the search for this treatment by patients with edentulism has been expanded. Even so, like any intervention in a living organism, the rehabilitation treatment through the use of implants presents possible complications, both in relation to the implants and the implant-supported rehabilitations. As much as the correct planning does not prevent the occurrence of these failures and complications in the implant-prosthetic treatment, it will allow the professional to predict these complications, enabling an efficient conduct in each case.

Descriptors: Dental Implants; Prostheses and Implants; Complications.

\section{Resumen}

Desde que se ha comprobado el uso sistemático de implantes como una posibilidad terapéutica eficaz, se ha ampliado la búsqueda de este tratamiento por parte de pacientes con edentulismo. Aun así, como toda intervención en un organismo vivo, el tratamiento de rehabilitación mediante el uso de implantes presenta posibles complicaciones, tanto en relación a los implantes como a las rehabilitaciones implantosoportadas. En tanto que una correcta planificación no evite la ocurrencia de estos fallos y complicaciones en el tratamiento implanto-protésico, permitirá al profesional predecir estas complicaciones, posibilitando una conducta eficiente en cada caso.

Descriptores: Implantes Dentales; Prótesis e Implantes; Complicaciones.

\section{INTRODUÇÃO}

A perda dentária é considerada um dos principais agravos à saúde bucal devido à alta prevalência e aos danos estéticos, funcionais, psicológicos e sociais que acarreta ${ }^{1}$. Os implantes osseointegráveis e as próteses dentárias implanto-suportadas têm sido a primeira opção para pacientes total ou parcialmente desdentados, em virtude de proporcionarem uma reabilitação com alta retenção, estabilidade e conforto ${ }^{2,3}$.

Assim como qualquer tratamento terapêutico, o sucesso dos implantes e das reabilitações implanto-suportadas dependem da observância rigorosa de critérios estabelecidos para garantir 0 bom prognóstico dos tratamentos ${ }^{4}$. Apesar de ter eficiência comprovada, a ocorrência de falhas dos implantes não é rara, podendo a taxa de insucesso variar entre $1,5 \%$ e $3,5 \%$, podendo chegar a até $10 \%$.

A introdução acidental intra-sinusal do implante, peri-implantite, hemorragia iatrogênica, danos a estrutura dos implantes, fratura ou mobilidade do implante, fratura mandibular, implantes em posição e angulação desfavorável, deiscência da ferida cirúrgica, infecção e distúrbios neurossensoriais são algumas das possíveis intercorrências em um tratamento reabilitador envolvendo implantes osseointegráveis, sendo de fundamental conhecimento aos profissionais da área ${ }^{7}$

Sendo assim, presente estudo tem como objetivo revisar a literatura a respeito de falhas e complicações na terapia de reabilitação com implantes osseointegráveis auxiliando o profissional na demanda clínica.

MATERIAL E MÉTODO

Foi realizada uma busca eletrônica, não sistemática, nas bases de dados Lilacs, Pubmed/Medline, Scielo e ScienceDirect, cruzando-se os seguintes descritores em língua inglesa e portuguesa: "Implant"; "Complications", "Prosthetic". Foram incluídos artigos de revisão de literatura, revisão sistemática, meta-análise, estudos clínicos randomizados, além de livros pertinentes ao assunto, publicados no período de 1982 a 2020.

\section{REVISÃO DA LITERATURA}

- Complicações em Implantodontia

A análise da literatura evidencia que a etiologia das falhas de implantes é multifatorial e que podem ser minimizadas com um correto e minucioso planejamento, além do preparo teórico e clínico do profissional ${ }^{8}$.

Alves et al, em 2017, dividiu as complicações em implantodontia em três grupos: as relacionadas com o plano de tratamento (fatores sistêmicos, angulação e localização incorreta dos implantes), as relacionadas com a anatomia local (lesão de 
nervos, fenestrações, complicações sinusais e infecções) e as relacionadas com o processo cirúrgico propriamente dito (superaquecimento ósseo, falta de estabilidade primária $)^{7}$.

O planejamento é uma etapa primordial do tratamento, no qual são colhidas uma gama de informações do quadro sistêmico, hábitos deletérios e história clínica. $\mathrm{O}$ aumento da idade (60-70 anos) mostrou forte associação com falha na reabilitação com implantes, bem como tabagismo, diabetes, radiação na cabeça e pescoço e uso de estrogênio pós-menopausa ${ }^{9}$. Ainda na fase de planejamento uma incorreta angulação mesio-distal, buco-lingual, ápicecoronal pode afetar a etapa protética do tratamento; a fim de prevenir essa ocorrência é indicado o uso de guias cirúrgicas para orientar as perfurações ${ }^{10}$.

$\mathrm{Na}$ fase transoperatória, lesões a nervos também são comuns e suas possíveis causas incluem: trauma e desenho deficiente do retalho, penetração do nervo durante a osteotomia, preparação e compressão do nervo pelo corpo do implante no canal $^{10}$. O nervo alveolar inferior é o mais lesionado $(64,4 \%)$, seguido pelo nervo lingual $(28,8 \%)$ durante procedimentos de cirurgia oral ${ }^{11}$. Se o canal mandibular não for visualizado corretamente no exame radiográfico, o ideal é que seja realizado tomografia computadorizada. Ellies \& Hawker, em 1993, encontraram uma sensação alterada incidência de $36 \%$, dos quais $10 \%$ a $15 \%$ desses pacientes nunca recuperaram sensação $^{12}$.

Quadros hemorrágicos apresentam uma incidência de $24 \%{ }^{13}$. Locais de risco em mandíbula incluem a fossa sublingual e córtex lingual. Dentro de 30 minutos, uma artéria rompida na área pode causar uma taxa de perda sanguínea de $14 \mathrm{ml} / \mathrm{min}$ e se mais de 500 $\mathrm{ml}$ de sangue perda ocorre, podendo resultar em hipotensão. Nesses casos, pressão extraoral das artérias submentual e submandibular contra o corpo da mandíbula por 20 minutos pode auxiliar na hemostasia ${ }^{10}$.

No estudo de Ardekin et al, em 2016, perfurações na membrana sinusal maxilar foram mais comumente encontradas em áreas com altura óssea mínima $(<5 \mathrm{~mm})$, mas isso não afetou a taxa geral de sucesso do implante ${ }^{14}$. Perder um implante no seio maxilar é relativamente incomum; contudo, nos casos com menos de $5 \mathrm{~mm}$ de osso, a mastigação pode fazer com que os implantes se movam durante a maturação do enxerto. Nesses casos o cirurgião deverá identificar os prós e contras de se realizar um tratamento com carga imediata $^{15}$.
A mandíbula é o osso da face mais frequentemente fraturado. Tentativas de colocar implantes em pacientes com mandíbulas severamente atróficas e o uso de implantes mais longos em sítios com subinstrumentação pode aumentar o risco de fratura $^{10}$. Aumento da temperatura durante a perfuração do osso pode causar necrose trazendo efeitos prejudiciais para osseointegração. Além disso, recomenda-se o uso de irrigação salina, pressão intermitente nas brocas (pausando a cada 3 a 5 segundos) e a utilização de brocas novas ${ }^{16}$.

- Complicações em Próteses Sobre Implante

As complicações protéticas relacionadas ao implante incluem: danos a estrutura de conexão do implante; assentamento inadequado do componente; fratura ou afrouxamento do parafuso de retenção e do componente, fratura da cerâmica de recobrimento ou da coroa e decimentação 8,17 .

Em um estudo clínico realizado entre 1992 e 2000, 677 pacientes receberam tratamento com implantes, as complicações identificadas como protéticas foram: fratura ou afrouxamento do pilar; necessidade de substituição de O-ring inferior em até 12 meses após a colocação da prótese; necessidade de ajuste oclusal ou protético da restauração definitiva após 2 semanas ou mais da entrega; e necessidade de recolocação de prótese fixa solta dentro de 2 semanas após a entrega. Foram associados a essas complicações fatores como: a localização do implante (anterior versus posterior); tipo de prótese (removível versus fixo); proximidade do implante; qualidade óssea; e angulação do pilar ${ }^{18}$.

De acordo com revisão sistematica de Jain et al. ${ }^{19}$, em 2018, a prótese cimentada mostra menos retenção e falhas em comparação com a prótese parafusada, a taxa de falha de retenção de restaurações até 5 anos e mais de 5 anos para coroas aparafusadas foi comparativamente superior ao das coroas cimentadas. Porém, ainda deve-se levar em conta os critérios para sua indicação.

Em relaçao a carga imediata ou tardia ambas demonstraram taxas de sobrevida semelhantes, sendo a qualidade óssea o fator mais importante. $\mathrm{O}$ diâmetro e o comprimento dos implantes não parecem influenciar os resultados ${ }^{20}$. Implantes com prótese unida ou não, não apresentaram evidências que sugeriram que a imobilização (união das coroas) reduz a perda óssea. Sendo a perda óssea decorrente principalmente da higiene deficiente. Mesmo unidas essas coroas protéticas devem oferecer meio de higiene eficiente ${ }^{21}$. 
DISCUSSÃO

$$
\text { Pjetursson et } \text { al. }^{22} \text {, em revisão }
$$

sistemática mostraram que até $38,7 \%$ de todos os implantes instalados em pacientes parcialmente desdentados tiveram algum tipo de complicação durante o período de observação de 5 anos. Segundo McDermott et $\mathrm{al}^{18}{ }^{18}$, em seu estudo, as complicações mais comumente observadas foram de origem inflamatória, em $10.2 \%$ dos casos, seguida por complicações protéticas, em $2.7 \%$ dos casos, e complicações na fase operatória, em $1.0 \%$ dos casos.

Já em um estudo realizado por Goodacre et al. ${ }^{13}$, em 2003, as complicações mais comuns dos implantes (aquelas com uma incidência superior a $15 \%$ ) foram o afrouxamento do mecanismo de retenção da prótese sobre implante (33\%), a perda de implantes em maxila (25\%), complicações relacionadas à hemorragia (24\%), fratura de resina com próteses parciais fixas (22\%), perda de implante com overdentures maxilares (21\%), overdentures precisando ser reembasados (19\%), perda de implante em osso tipo IV (16\%) e fratura de clip e fixação de overdenture (16\%).

Akpinar et al. ${ }^{23}$ em 2000, concluiram em seu estudo que o sucesso de uma reconstrução com implante não depende somente do implante ou de seus dentes vizinhos. A influência da reconstrução suportada por implantes e os dentes naturais antagonistas também podem ser importantes para o sucesso do tratamento. Para os autores, as tensões geradas em torno do corpo rígido do implante e entre um dente antagonista flexível são importantes para a seleção do implante e que um implante pode ser responsável por intrusões em dentes antagonistas naturais.

CONCLUSÃO

As complicações na implantodontia podem ocorrer nas diversas fases do tratamento. Um planejamento adequado deve abranger todas as fases (operatória, inflamatória, protética e precoce e tardia) e ainda as condições do profissional e paciente. Por mais que o correto planejamento não impeça a ocorrência dessas falhas e das complicações no tratamento implantoprotético, permitirá ao profissional uma previsibilidade desses intercorrências, possibilitando uma conduta eficiente a cada caso.

\section{REFERÊNCIAS}

1. Brasil. SB Brasil 2010: Pesquisa Nacional de Saúde Bucal: resultados principais / Ministério da Saúde. Secretaria de Atenção à Saúde. Secretaria de Vigilância em Saúde. - Brasília: Ministério da Saúde, 2012.
2. Corbella S, Del Fabbro M, Taschieri S, De Siena F, Francetti L. Clinical evaluation of an implant maintenance protocol for the prevention of periimplant diseases in patients treated with immediately loaded full-arch rehabilitations. Int $\mathrm{J}$ of Dant Hyg. 2011; 9(3):216-22.

3. Livio EM, Silva RS, Poluha RL. Higienização e manutenção de prótese total fixa sobre implante. Arch Health Invest. 2019;8(7):390-3.

4. Kracher CM, Smith WS. Oral health maintenance dental implants. Dent Assist. 2010;79(2):27-35.

5. Friberg B, Jemt T, Lekholm U. Early failures im 4641 consecutively placed Branemark dental implants: a study from stage 1 surgery to the connection of completed prostheses. Int $\mathrm{J}$ Oral Maxillofac Implants. 1991;6:142-6.

6. Jung RE, Pjetursson BE, Glauser R, Zembic A, Zwahlen M, Lang NP. A systematic review of the 5-year survival and complication rates of implant-supported single crowns. Clin. Oral Impl. Res. 2008;19(2):119-30.

7. Alves LMN, Hidalgo LRC, Conceição LS, Oliveira GM, Borges KRF, Passos WG. Implants complications:a literature review. J Orofac Invest. 2017;4(1):20-9.

8. Ramalho-Ferreira G, Faverani LP, Gomes PCM, Assunção WG, Garcia Junior IR. Complicações na reabilitação bucal com implantes osseointegravéis. Revista Odontológica de Araçatuba. 2010;31(1):51-5.

9. Moy PK, Medina D, Shetty V, Aghaloo TL. Dental implant failure rates and associated risk factors. Int J Oral Maxilliofac Implants. 2005;20(4):569-77.

10. Misch K, Hom-Lay W. Implant Surgery Complications: Etiology and Treatment. Implant dentistry. 2008;17:159-68.

11. Tay $A B$, Zuniga JR. Clinical characteristics of trigeminal nerve injury referrals to a university centre. Int $J$ Oral Maxillofac Surg. 2007;36(10):922-7.

12. Ellies LG, Hawker PB. The prevalence of altered sensation associated with implant surgery. Int J Oral Maxillofac Implants. 1993; 8(6):674-9.

13. Goodacre CJ, Bernal G, Rungcharassaeng K, Kan JY. Clinical complications with implants and implant prostheses. J Prosthet Dent. 2003; 90(2):121-32.

14. Ardekian L, Oved-Peleg E, Mactei EE, Peled M. The clinical significance of sinus membrane perforation during augmentation of the maxillary sinus. J Oral Maxillofac Surg.2006;64(2):277-82.

15.Peleg M, Garg AK, Mazor Z. Predictability of simultaneous implant placement in the severely atrophic posterior maxilla: A 9-year longitudinal experience study of 2,132 implants placed into 731 human sinus grafts. Int J Oral Maxillofac Implants. 2006;21:94-102. 
16. Ericksson RA, Albrektsson T. Temperature threshold levels for heat-induced bone tissue injury: A vital-microscopic study in the rabbit. $\mathrm{J}$ Prosthet Dent. 1983;50:101-7.

17. Sadid-Zadeh R, Kutkut A, Kim H. Prosthetic failure in implant dentistry. Dent Clin North Am. 2015;59:195-214.

18. McDermott NE, Chuang SK, Woo VV, Dodson TB. Complications of dental implants: identification, frequency, and associated risk factors. Int $\mathrm{J}$ Oral Maxillofac Implants. 2003;18(6):848-55.

19. Jain JK, Sethuraman R, Chauhan S, Javiya P, Srivastava S, Patel R, Bhalani B. Retention failures in cement- and screw-retained fixed restorations on dental implants in partially edentulous arches: A systematic review with meta-analysis. J Indian Prosthodont Soc. 2018; 18(3):201-11.

20. Muelas-Jiménez MI, Olmedo-Gaya MV, Manzano-Moreno FJ, Reyes-Botella C, Vallecillo-Capilla M. Long-Term Survival of Dental Implants with Different Prosthetic Loading Times in Healthy Patients: A 5-Year Retrospective Clinical Study. J Prosthodont. 2017;26(2):99-106.

21. Ravidà A, Saleh MHA, Muriel MC, Maska B, Wang HL. Biological and technical complications of splinted or nonsplinted dental implants: a decision tree for selection. Implant Dent. 2018;27(1):89-94.

22. Pjetursson BE, Sailer I, Zwahlen M, Hämmerle $\mathrm{CH}$. A systematic review of the survival and complication rates of all-ceramic and metalceramic reconstructions after an observation period of at least 3 years. Part I: Single crowns. Clin Oral Implants Res. 2007;18 Suppl 3:73-85. Erratum in: Clin Oral Implants Res. 2008; 19(3):326-8.

23. Akpinar I, Anil N, Parnas L. A natural tooth's stress distribution in occlusion with a dental implant. J Oral Rehabil. 2000;27(6):538-45.

\section{CONFLITO DE INTERESSES}

Os autores declaram não haver conflitos de interesse

\section{AUTOR PARA CORRESPONDÊNCIA}

\section{Rodrigo Lorenzi Poluha}

Departamento de Odontologia,

Universidade Estadual de Maringá -UEM

Avenida Mandacaru, $n^{\circ}$ 1.550. Vila Santa Izabel

87080-000 Maringá-PR, Brasil

E-mail: rodrigopoluha@gmail.com 